\title{
Relation between neuropsychological impairment and functional disability in patients with chronic fatigue syndrome
}

Christopher Christodoulou, John DeLuca, Gudrun Lange, Susan K Johnson, Sue Ann Sisto, Leo Korn, Benjamin H Natelson

\begin{abstract}
Objectives-To examine the relation between neuropsychological impairment and functional disability in patients with chronic fatigue syndrome, and determine whether the relation is independent of psychiatric factors.

Methods-The subjects were 53 patients with chronic fatigue syndrome and 32 healthy controls who did not exercise regularly. Subjects were administered a structured psychiatric interview and completed questionnaires focusing on depression and functional disability. They also completed a battery of standardised neuropsychological tasks focusing on the cognitive domains that patients with chronic fatigue syndrome experience as particularly difficult: memory (verbal and visual), and attention/concentration. A test score was defined as failing when it was $\geqslant 2$ SD below the mean of the healthy controls after controlling for demographic factors.
\end{abstract}

Results-Those patients with chronic fatigue syndrome with higher numbers of failing neuropsychological test scores reported significantly more days of general inactivity in the past month than those with fewer failing scores. This result remained significant even after partialling out the contribution of the presence of a comorbid axis I psychiatric episode and the overall level of depressive symptomology. Patients with failing verbal memory scores were particularly functionally disabled compared with those with passing scores.

Conclusion-A relation was found between cognitive impairment and functional disability which could not be explained entirely on the basis of psychiatric factors.

(F Neurol Neurosurg Psychiatry 1998;64:431-434)

Keywords: chronic fatigue syndrome; cognition; psychiatric illness

Correspondence to: Dr John DeLuca, Kessler Institute for Rehabilitation, Neuropsychology and Neuroscience Research Laboratory, Research Department, 1199 Pleasan Valley Way, West Orange,

New Jersey 07052, USA.

Received 2 April 1997 and in final revised form 4

December 1997

Accepted 9 December 1997
Regardless of its aetiology, chronic fatigue syndrome can have a devastating impact on the functional ability of such patients to carry out their daily activities. ${ }^{7-9}$ For example, a prevalence study found that $43 \%$ of patients with chronic fatigue syndrome were unable to work or attend school. ${ }^{7}$ Neuropsychological complaints are also prominent in chronic fatigue syndrome. Up to $85 \%$ of patients report cognitive impairment, often in the areas of concentration and memory. ${ }^{10}$ These complaints are troubling and patients consider them to be among the most relevant factors leading to their social and occupational dysfunction. ${ }^{11}$ Some recent studies have substantiated subtle cognitive deficits in such patients, tending to cluster in the areas of concentration, complex information processing, and memory. ${ }^{12-15}$

Whereas functional disability and neuropsychological impairment have both been documented in the chronic fatigue syndrome population, a recent article outlined the need to examine the possible association between performance in these two domains. ${ }^{16}$ If chronic fatigue syndrome follows the pattern displayed in other disorders such as multiple sclerosis and human immunodeficiency virus type 1 (HIV) then cognitive and functional impairment should be positively correlated. ${ }^{17} 18$

However, one important consideration in assessing such a relation is the possibility that functional status and neuropsychological performance could both be affected by psychiatric illness. Depression has been a particular concern of many people working on chronic fatigue syndrome. This concern has arisen in part because of symptoms such as fatigue and somatic complaints shared by both disorders, ${ }^{11}$ although it should be noted that the pattern of symptoms in patients with chronic fatigue syndrome with concurrent depression has been found to differ from that of depressed persons without the syndrome. ${ }^{19}$ If chronic fatigue syndrome actually represents a form of psychopathology (for example, an affective disorder), then it could be hypothesised that the psychiatric disorder may be responsible for both the cognitive deficits and functional impairment found in this population. The present study therefore included measures of psychiatric variables to statistically control for their possible effects on cognition and functional abilities.

The purpose of the present study was to examine the relation between cognitive performance and functional status in patients with chronic fatigue syndrome. It was 
hypothesised that those patients who failed more neuropsychological tasks would display greater functional disability. Furthermore, this relation was expected to remain significant even when the presence of psychiatric symptoms was taken into account.

\section{Subjects and methods}

SUBJECTS

The present study consisted of 32 healthy controls and 53 patients with chronic fatigue syndrome. Both groups consisted primarily of women (controls 90.6\%; chronic fatigue syndrome $88.7 \%$ ) with a mean age in the mid-thirties (control mean age 37.3 (SD 9.8); chronic fatigue syndrome mean 36.1 (SD 8.7)), and about 15 years of education (controls mean 15.5 (SD 2.2)); chronic fatigue syndrome mean 15.1 (SD 2.3)). Control subjects were recruited from local communities and colleges and were paid for their participation. Patients with chronic fatigue syndrome were either referred by themselves or physicians to the Chronic Fatigue Syndrome Center at UMDNJ-New Jersey Medical School.

All patients with chronic fatigue syndrome fulfilled the original United States Centers for Disease Control (CDC) case definition ${ }^{12}$ as well as the following additional inclusionary criteria: (1) chronic fatigue syndrome symptoms of at least moderate severity at the time of intake; (2) an illness duration of no more than four years (reducing the likelihood of symptoms that might arise secondarily from the psychosocial consequences associated with chronic illness; (3) an absence of psychiatric disorder in the five years before chronic fatigue syndrome onset, based on DSM-III-R criteria ${ }^{20}$ as measured by the diagnostic interview schedule (Q-DIS). ${ }^{21}$ The last criterion essentially eliminated patients with chronic fatigue syndrome with a history of psychiatric disorders before the onset of the syndrome, with the exception of one patient who had experienced a depressive episode many years earlier. Based on our experience, about $10 \%$ of our patients were excluded due to an axis 1 disorder in the five years before diagnosis. These additional criteria were used in an effort to improve the homogeneity of the pool of patients with chronic fatigue syndrome. ${ }^{22}$

All subjects received a complete history and physical examination at the Chronic Fatigue Syndrome Center. Potential subjects were excluded from both chronic fatigue syndrome and control groups if they had a history of bipolar affective disturbance, eating disorder, schizophrenia, or substance misuse as measured on the Q-DIS. Subjects were also excluded from both groups if they had a loss of consciousness for more than five minutes or engaged in a regular exercise regimen (this was used primarily to exclude controls who regularly exercise and hence control for the fact that patients with chronic fatigue syndrome are typically inactive due to their illness).

METHODS AND PROCEDURES

Functional disability was examined using a modified version of the functional status ques- tionnaire (FSQ $)^{23}$ which was designed to measure activity over the preceding month. Three aspects of functional impairment were assessed: activities of daily living, social activities, and general inactivity. Activities of daily living was measured by combining the basic and intermediate activities of daily living subscales. Social activities were measured by combining the subscales of frequency of social activities and difficulties in social activities. Finally, general inactivity was assessed by summing days in bed and days cut down, measures of a general reduction in activity irrespective of the type of activity involved. In our modification of the FSQ, days in bed were not counted when calculating days cut down in the past month, thus scores on the two subscales were expected to be relatively independent of one another. This was empirically verified by examining their correlation with one another $(r=-0.192$, $\mathrm{p}=0.170)$. Therefore, the sum of these two variables provided an overall measure of the number of days of general inactivity over the past month. Such a reduction in activity is a major CDC criterion for the diagnosis of chronic fatigue syndrome.$^{1-3}$

Two psychiatric factors were considered in the present study. The first was the severity of depressive symptomology, using the Beck depression inventory (BDI) ${ }^{24}$ The mean BDI total score for patients with chronic fatigue syndrome placed them in the mildly depressed range (mean 13.1 (SD 7.1)). The second factor was the presence or absence of a DSM-III-R axis I psychiatric diagnosis, categorised dichotomously on the basis of the Q-DIS. Using the Q-DIS two groups of patients with chronic fatigue syndrome were formed, one which did $(47 \%)$ and one which did not have a concurrent axis I disorder (53\%). The most common diagnosis was major depression, found in $84 \%$ of those with an axis I disorder. Other diagnoses included dysthymia (16\%), generalised anxiety disorder (20\%), panic disorder $(20 \%)$, and phobia $(20 \%)$ (the sum total exceeds $100 \%$ because some patients received more than one diagnosis).

The neuropsychological test battery consisted of instruments sensitive to the cognitive domains found to be impaired in patients with chronic fatigue syndrome-memory and attention/concentration. Three scores from the California verbal learning test (CVLT) were used to measure verbal memory: total score trials one through five, short delay free recall, and long delay free recall. ${ }^{25}$ Visual memory was measured by immediate and delayed recall on the Rey-Osterreith complex figure (ROCF). ${ }^{26}{ }^{27}$ Attention and concentration were measured with the total score from the paced auditory serial addition test (PASAT) ${ }^{28}$ and forward and backward digit span from the Wechsler adult intelligence scale revised (WAIS-R). ${ }^{29}$

To measure cognitive dysfunction among the patients with chronic fatigue syndrome, their performance on the neuropsychological tests was compared with that of the healthy controls. The raw scores of both groups were converted to standardised residual scores to control for 


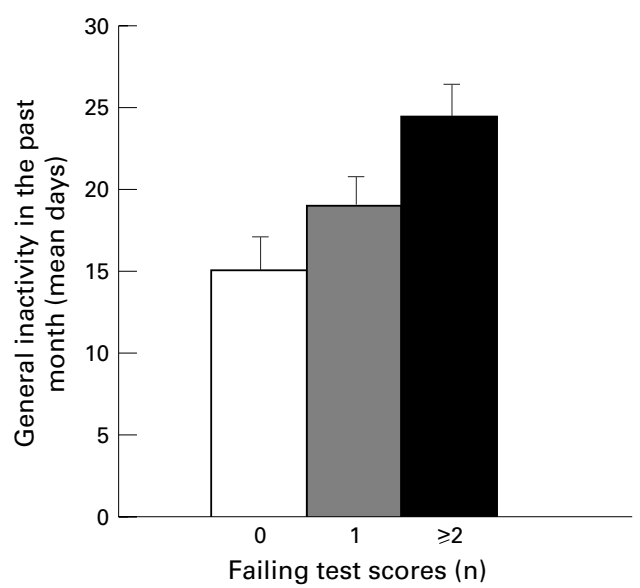

Mean (SE) days of general inactivity over the past month among patients with chronic fatigue syndrome with zero $(n=19)$, one $(n=20)$, or two or more $(n=14)$ failing neuropsychological test scores.

the possible effects of demographic variables, using a technique similar to that of Rao et al. ${ }^{30}$ Specifically, the demographic variables of age and education were regressed on each of the neuropsychological test scores. A product of these analyses, the standardised residual score, represents the difference between a subject's predicted (on the basis of age and education data from the healthy control subjects) and actual test scores, and indicates that portion of an individual persons score which cannot explained by the demographic variables. A patient with chronic fatigue syndrome was considered to have a failing test score when the residual score was $\geqslant 2$ SD below that of the normal controls. The failing test scores were used to quantify levels of cognitive impairment that would be expected to have some clinical significance for the functional abilities of patients with chronic fatigue syndrome. A total score was calculated which summed the number of failed test scores that a patient accumulated. Patients with chronic fatigue syndrome were then classified into three groups based on their number of failing test scores $(0,1$, or $\geqslant 2)$. In addition, for each of the three domains of verbal memory, visual memory, and attention/concentration, subjects were dichotomously divided into those with and those without one or more failing test scores.

\section{Results}

The primary hypothesis was that those patients with chronic fatigue syndrome who failed more neuropsychological tasks would display greater functional disability. Analysis of variance (ANOVA) for general inactivity resulted in significant differences between subjects on the basis of the number of tests failed $(F(2,50)=4.959, \mathrm{p}=0.011$, figure $)$. Post hoc Tukey tests were then applied to the general inactivity data to examine pairwise comparisons between the groups. The only significant finding was that those who failed two or more tests reported significantly more days of inactivity in the past month (mean 24.29 (SD 7.22)) than those who did not fail any tests (mean 15.00 (SD 8.97)) ( $\mathrm{p}=0.008$ ). ANOVAs for activities of daily living and for social activities resulted in no significant differences due to tests failed.

The next step was to specify the area(s) of cognition most related to general inactivity. A $t$ test disclosed that those patients who had one or more failing verbal memory test score had significantly higher general inactivity scores than those who did not fail such tests $(t 1,51)=-3.00, \mathrm{p}=0.004)$, averaging 25 (mean 24.96 (SD 6.81)) versus 17 (mean 16.95 (SD 8.79 )) days of general inactivity in the past month. $t$ Tests resulted in no significant differences in general inactivity due to visual memory failures or attention and concentration failures.

Another hypothesis of the present study was that the relation between cognitive deficit and functional impairment would remain significant even when the presence of psychiatric symptoms was taken into account. To control for the possible contribution of the psychiatric variables to the relation between cognition and functional status, the general inactivity data were reanalysed by analysis of covariance (ANCOVA) with tests failed as the between group factor, and both BDI total score and presence of axis I disorder as covariates. Group differences remained significant for tests failed $(F(2,48)=4.441, \mathrm{p}=0.017)$, indicating that the psychiatric variables could not fully account for the finding that those performing worse on the neuropsychological tasks were also more functionally impaired.

Additional analyses examined the relation between each of the psychiatric variables (BDI and axis I disorder) with general inactivity and tests failed. The presence or absence of an axis I disorder was not associated with differences in either general inactivity ( $t$ test), nor tests failed (Mann-Whitney test). BDI total score was significantly positively correlated with general inactivity (Pearson's $r=0.375, \mathrm{p}=0.006$ ). However, BDI total score was not associated with tests failed (ANOVA).

\section{Discussion}

To our knowledge the present study is one of the first to examine the relation between cognitive impairment and functional disability in patients with chronic fatigue syndrome. ${ }^{31}$ The relative lack of previous research on this topic is noteworthy as many patients consider these cognitive symptoms to be the source of some of their greatest frustration and disability. ${ }^{116}$ The present results show that patients with chronic fatigue syndrome who perform worse on neuropsychological testing are also more likely to display greater functional disability in their daily activities. More specifically, those patients with chronic fatigue syndrome with poorer word list acquisition and free recall on the CVLT were also those who were most functionally disabled in their daily lives. Whereas most patients in the current chronic fatigue syndrome sample reported substantial levels of general inactivity over the past month, those with failing scores on the CVLT reported even greater inactivity. 
The results further show that the association between cognitive impairment and functional disability was not simply a consequence of the psychiatric factors. Differences in neuropsychological impairment were relevant to functional status, even after removing the variance associated with both the presence of an axis I diagnosis and the severity of depressive symptomology. It is possible that the methods used to measure psychopathology were not entirely adequate in capturing the full range of potential psychopathology. This inadequacy may have lessened their overall contribution to the explanation of functional impairment. Thus more sensitive measures of psychopathology may have been able to account for more of the variability in functional status than was found in the present study.

The association of neuropsychological deficits with functional impairment in patients with chronic fatigue syndrome is similar to findings obtained among other medical populations such as those with $\mathrm{HIV},{ }^{17}$ multiple sclerosis, ${ }^{18}{ }^{32}$ Alzheimer's disease, ${ }^{33}$ and stroke. $^{34}$ Neuropsychological assessment is a valuable clinical tool in the assessment and treatment of various disorders because cognitive impairment has functional implications for the lives of patients, and can help predict future likelihood of disability. ${ }^{32}{ }^{34}$ The cross sectional nature of the present study precludes the conclusion that cognitive deficits cause functional impairment in chronic fatigue syndrome. None the less, this study provides empirical evidence that such a relation could exist among a subset of patients with chronic fatigue syndrome. Future studies should examine whether cognitive deficits in newly diagnosed patients with chronic fatigue syndrome can predict long term functional disability.

It should be noted that the present results are based on a rather selective sample of patients with chronic fatigue syndrome. Patients in the present study had moderate to severe chronic fatigue symptoms and had no history of psychiatric disorder before onset of the syndrome. It is not clear whether the relation between cognition and functional disability would generalise to the broader population of patients with chronic fatigue syndrome.

This study was supported in part by grants R01-H52810A and AI-32247 and from the National Institutes of Health.

1 Holmes GP, Kaplan JE, Gantz NM, et al. Chronic fatigue syndrome: a working case definition. Ann Intern Med 1988;108:387-9.

2 Schluederberg A, Straus SE, Peterson P, et al. Chronic fatigue syndrome research: definition and medical outcome assessment. Ann Intern Med 1992;117:325-31.

3 Fukuda K, Straus SE, Hickie I, et al. The chronic fatigue syndrome: a comprehensive approach to its definition and syndrome: a comprehensive approach to

4 Komaroff AL. Chronic fatigue syndromes: relationship to chronic viral infections. F Virol Methods 1988;21:3-10.

5 Strober W. Immunological function in chronic fatigue syndrome. In: Straus SE, ed. Chronic fatigue syndrome. New York: Marcell Dekker, 1994:207-37.

6 Manu P, Lane TJ, Matthews DA. Chronic fatigue syndromes in clinical practice. Psychother Psychosom 1992 58:60-8.
7 Lloyd AR, Hickie I, Boughton CR, et al. Prevalence of chronic fatigue syndrome in an Australian population. Med f Aust 1990;153:522-8.

8 Natelson BH, Johnson SK, DeLuca J, et al. Reducing heterogeneity in chronic fatigue syndrome: a comparison with depression and multiple sclerosis. Clin Infect Dis 1995; 21:1204-10.

9 Vercoulen JHMM, Swanink CMA, Fennis JFM, et al. Dimensional assessment of chronic fatigue syndrome. $\mathcal{f}$ Psychosom Res 1990;34:383-92.

10 Komaroff AL. Clinical presentation of chronic fatigue syndrome. In: Bock GR, Whelan J, eds. Chronic fatigue syndrome. Ciba Found Symp 1993;173:43-61.

11 Abbey SE, Garfinkel PE. Chronic fatigue syndrome and depression: cause, effect, or covariate. Reviews of Infectious Diseases 1991;13(suppl 1):S73-83.

12 DeLuca J, Johnson SK, Natelson BH. Information processing in chronic fatigue syndrome and multiple sclerosis. Arch Neurol 1993;50:301-4.

13 DeLuca J, Johnson SK, Beldowicz D, et al. Neuropsychological impairments in chronic fatigue syndrome, multiple sclerosis, and depression. I Neurol Neurosurg Psychiatry 1995;58:38-43.

14 Joyce E, Blumenthal S, Wessely S. Memory, attention, and executive function in chronic fatigue syndrome. 7 Neurol Neurosurg Psychiatry 1996;60:495-503.

15 Michiels V, Cluydts R, Fischler B, et al. Cognitive functioning in patients with chronic fatigue syndrome. F Clin Exp Neuropsychol 1996;18:666-7.

16 Moss-Morris R, Petrie KJ, Large RG, et al. Neuropsychological deficits in chronic fatigue syndrome: artifact or reality? F Neurol Neurosurg Psychiatry 1996;60:474-7.

17 Heaton RK, Marcotte TD, White DA, et al. Nature and vocational significance of neuropsychological impairment associated with HIV infection. Clinical Neuropsychologist 1996;10:1-14

18 Rao SM, Leo GJ, Ellington L, et al. Cognitive dysfunction in multiple sclerosis. II. Impact on employment and social functioning. Neurology 1991;41:692-6.

19 Johnson SK, DeLuca J, Natelson BH. Depression in fatiguing illness: comparing patients with chronic fatigue syndrome, multiple sclerosis and depression. 7 Affect Disord 1996;39:21-30

20 American Psychiatric Association. Diagnostic and statistical manual of mental disorders, 3rd ed revised. Washington, DC: APA, 1987

21 Marcus S, Robins LN, Bucholz K. Quick diagnostic interview schedule III-R, version 1. St Louis, MO: Washington University School of Medicine, 1990.

22 Christodoulou C, DeLuca J, Lange G, et al. Efforts to reduce heterogeneity in chronic fatigue syndrome research. In: Mostofsky DI, Yehuda S, eds. Chronic fatigue syndrome. NY: Plenum Press, 1997:73-94.

23 Jette AM, Davies AR, Cleary PD, et al. The functional status questionnaire: reliability and validity when used in primary care. $\mathcal{F}$ Gen Intern Med 1986;1:143-9.

24 Beck AT. Beck depression inventory. San Antonio, TX: The Psychological Corporation, 1987.

25 Delis DC, Kramer JH, Kaplan E, et al. California verbal learning test: Adult version. San Antonio, TX: The Psychological Corporation, 1987.

26 Osterreith PA. Le test de copie d'une figure complexe. Arch Psychol 1944;30:206-356. Translated by Corwin J, Bylsma FW. Clinical Neuropsychologist 1993;7:9-15.

27 Rey A. Psychological examination of traumatic encephalopathy. Arch Psychol 1941;28:286-340. Sections translated by Corwin J, Bylsma FW, Clinical Neuropsychologist 1993;7: 4-9.

28 Brittain JL, La Marche JA, Reeder KP, et al. Effects of age and IQ on paced auditory serial addition task (PASAT) performance. Clinical Neuropsychologist 1991;5:163-75.

29 Wechsler D. WAIS-R manual. New York: The Psychological Corporation, 1981.

30 Rao SM, Leo GJ, Bernardin L, et al. Cognitive dysfunction in multiple sclerosis. I. Frequency, patterns, and prediction. Neurology 1991;41:685-91.

31 Lutgendorf S, Klimas NG, Antoni M, et al. Relationships of cognitive difficulties to immune measures, depression, and illness burden. Fournal of Chronic Fatigue Syndrome 1995;2: 23-41.

32 Amato MP, Ponziani G, Pracucci G, et al. Cognitive impairment in early-onset multiple sclerosis: pattern, predictors, and impact on everyday life in a 4-year follow-up. Arch Neurol 1995;52:168-72.

33 Fitz AG, Teri L. Depression, cognition, and functional ability in patients with Alzheimer's disease. $\mathcal{F} \mathrm{Am}$ Geriatr Soc 1994;42:186-91.

34 Tatemichi TK, Desmond DW, Stern Y, et al. Cognitive impairment after stroke: frequency, patterns, and relationship to functional abilities. $\mathcal{F}$ Neurol Neurosurg Psychiatry 1994;57:202-7. 